\title{
Age dependency of risk factors for cognitive decline
}

\author{
N. Legdeur ${ }^{1 *}$ (D, M. W. Heymans², H. C. Comijs ${ }^{3}$, M. Huisman 2,4 , A. B. Maier ${ }^{5,6}$ and P. J. Visser ${ }^{1,7}$
}

\begin{abstract}
Background: Risk factors for cognitive decline might depend on chronological age. The aim of the study was to explore the age dependency of risk factors for cognitive decline in cognitively healthy subjects aged 55-85 years at baseline.

Methods: We included 2527 cognitively healthy subjects from the Longitudinal Aging Study Amsterdam (LASA). Median follow-up was 9.1 (IQR: 3.2-19.0) years. The association of genetic and cardiovascular risk factors, depressive symptoms, inflammation markers and lifestyle risk factors with decline in MMSE and memory function was tested using spline regression analyses.
\end{abstract}

Results: Subjects were on average 70.1 (SD 8.8) years old at baseline. Based on a spline regression model, we divided our sample in three age groups: $\leq 70$ years (young-old), $>70-80$ years (old) and $>80$ years (oldest-old). The association of LDL cholesterol, homocysteine, hypertension, history of stroke, depressive symptoms, interleukin-6, a1-antichymotrypsin, alcohol use and smoking with cognitive decline significantly differed between the age groups. In general, the presence of these risk factors was associated with less cognitive decline in the oldest-old group compared to the young-old and old group.

Conclusions: The negative effect of various risk factors on cognitive decline decreases with higher age. A combination of epidemiological factors, such as the selection towards healthier subjects during follow-up, but also risk factor specific features, for example ensuring the cerebral blood flow in case of hypertension, explain this diminished association at higher age. It is important to take these age differences into account when applying preventive strategies to avert cognitive decline.

Keywords: Cognitive decline, Risk factors, Aging, Oldest-old

\section{Background}

Dementia is a growing health problem with an expected number of 115 million cases worldwide in 2050 [1]. The prevalence of dementia increases steeply with age from a prevalence of $2.6 \%$ in subjects aged $65-69$ years and a prevalence of $43.1 \%$ in subjects aged 90 years and older [1]. Insight in the risk factors for cognitive decline is essential in the search for preventive strategies for cognitive impairment and dementia. Former studies identified a range of potential risk factors including the APOE (apolipoprotein E) $\varepsilon 4$ allele, cardiovascular risk factors, depressive symptoms, inflammation markers and

\footnotetext{
* Correspondence: n.legdeur@vumc.nl

${ }^{1}$ Alzheimer Center Amsterdam, Department of Neurology, Amsterdam Neuroscience, Vrije Universiteit Amsterdam, Amsterdam UMC, PO Box 7057, 1007, MB, Amsterdam, the Netherlands

Full list of author information is available at the end of the article
}

lifestyle factors [2-8]. However, whether the effect of risk factors on cognitive decline in cognitively healthy subjects is dependent on age is not clear, as the majority of the previous studies did not discriminate between younger and older subjects and the number of subjects aged 80 years and older in these studies was generally low $[8,9]$. Still, there is increasing evidence that the association of risk factors with cognitive decline becomes less strong at higher age and may even have a protective effect [10]. For example, the risk of the APOE $\varepsilon 4$ allele on Alzheimer's dementia (AD) decreases after the age of 70 years [11]. In addition, the association of cardiovascular risk factors with cognitive decline might decrease with increasing age $[10,12-14]$.

The aim of the present study was to explore whether the association of risk factors with cognitive decline in

(c) The Author(s). 2018 Open Access This article is distributed under the terms of the Creative Commons Attribution 4.0 International License (http://creativecommons.org/licenses/by/4.0/), which permits unrestricted use, distribution, and 
cognitively healthy subjects across the age range of 55 to 85 years was dependent on age. We hypothesized that predictive accuracy would change with age for APOE $\varepsilon 4$ allele and cardiovascular risk factors based on previous studies and we performed exploratory analyses to test whether age effects were also present for other established risk factors including depressive symptoms, inflammation markers, alcohol use, smoking and physical activity.

\section{Methods}

\section{Study sample}

Data were derived from the on-going Longitudinal Aging Study Amsterdam (LASA) [15]. This is a longitudinal, population-based study in the Netherlands focusing on trajectories of physical, psychological, social and cognitive functioning in subjects aged 55 years and older. In 19921993 a random sample of men and women aged 5585 years, stratified for age and sex, from three geographic areas of the Netherlands (Amsterdam, Zwolle and Oss) was included. Follow-up measurements were conducted about every 3 years. Data collection included a main and medical interview conducted in the homes of the subjects. The main interview was done by trained and supervised interviewers and the medical interview was performed by trained nurses. All subjects gave informed consent and the study was approved by the Ethical Review Board of the VU University Medical Center (VUmc), Amsterdam, the Netherlands and conducted according to the principles of the Helsinki declaration.

At the start of the study in 1992-1993, 3107 subjects were enrolled. To select cognitively healthy subjects at baseline, subjects with an age and education corrected MMSE lower than 27 points were excluded (this cut-off is based on the lowest 10th percentile of the MMSE in the Maastricht Aging Study (MAAS) [16], leaving 2527 subjects at baseline. In 1995-1996, 2545 subjects were re-examined. See for further details about the following cycles of this LASA cohort and for the sample size per risk factor Additional file 1: Table S1 and S2.

\section{Measurements}

\section{Biomaterial}

The ApoE phenotyping was done either in 1992-1993 or 1995-1996 at the Immunochemisch Laboratorium of the VUmc. The blood samples were frozen at $-80{ }^{\circ} \mathrm{C}$ until determination in 1997-1998. The method used is described by Havekes et al. (1987) and consisted of isoelectric focusing of delipidated serum samples, followed by immunoblotting [17]. In the analyses, we used the presence of an ApoE $\varepsilon 4$ isoform (phenotypes $\varepsilon 2 / 4, \varepsilon 3 / 4$, $\varepsilon 4 / 4)$ as a dichotomous variable [17]. The ApoE $\varepsilon 4$ isoform was used as proxy for the presence of an APOE $\varepsilon 4$ allele.
Cholesterol levels (total cholesterol, High-Density Lipoprotein (HDL) cholesterol and Low-Density Lipoprotein (LDL) cholesterol) and homocysteine (in combination with vitamin B12) were determined in morning blood samples collected in 1995-1996 (second LASA cycle). Subjects were allowed to eat toast and drink tea, but no dairy products. The EDTA plasma samples were stored at $-80{ }^{\circ} \mathrm{C}$ and analyzed by the Department of Clinical Chemistry of the VUmc in 2001/2002 (homocysteine) and 2005 (cholesterol). For determination of total cholesterol and HDL cholesterol enzymatic colorimetric tests were used. LDL cholesterol was calculated as total cholesterol minus HDL-cholesterol minus VLDL-cholesterol; VLDL-cholesterol was calculated as total triglyceride concentration expressed in $\mathrm{mmol} / \mathrm{L}$ multiplied by 0.456 [18]. This method is less reliable when the triglyceride level is $\geq 5.0 \mathrm{mmol} / \mathrm{L}$. Therefore, this analysis was only done for triglyceride levels of < $5.0 \mathrm{mmol} / \mathrm{L}$. Total homocysteine was determined with the Abbott IMx analyser which uses fluorescence polarization immunoassay (FPIA) technology. Serum levels of vitamin B12 were determined at the Endocrine Laboratory of the VUmc with a competitive immunoassay luminescence on the automated ACS 180 System (Bayer Diagnostics, Mijdrecht, The Netherlands).

For determination of the inflammation markers (interleukin-6 (IL-6), C-reactive protein (CRP) and a1-antichymotrypsin (ACT)) serum collected in 19921993 (only in Amsterdam and Zwolle) was stored at $-80{ }^{\circ} \mathrm{C}$ until determination in 2002-2004. Sensitive regular immunoassays (ELISA) were used at Sanquin Research (Amsterdam) to determine IL-6, CRP and ACT. CRP was expressed in $\mathrm{ug} / \mathrm{ml}$, IL- 6 in $\mathrm{pg} / \mathrm{ml}$ and ACT in \% of normal plasma. The normal human plasma pool (\% NHP) used as a standard for ACT contained $\sim 300 \mathrm{mg}$ ACT per L. For part of the subjects, CRP levels were determined directly after blood sampling.

Both the cholesterol as the inflammation markers, were added to the analyses as continuous variables.

\section{Comorbidity}

Hypertension was defined as a blood pressure $>140 /$ $90 \mathrm{mmHg}$ measured at the upper arm or the use of antihypertensive medication collected in the first follow-up measurement in 1995-1996 (at baseline blood pressure was measured only at the finger). Post-hoc we also analyzed the association of a measured high blood pressure and the use of antihypertensive medication with cognitive decline separately.

The presence of a history of myocardial infarction (MI), DM or stroke was assessed by self-report. The assessment of comorbidity by self-report was found to be 
comparable with the medical information reported by the general practitioner [19].

Depressive symptoms were assessed using the Center for Epidemiologic Studies Depression scale (CES-D) [20]. The CES-D is a self-report scale containing 20 items describing depressive symptoms. The maximum score is 60 with higher scores indicating more depressive symptoms. In the analyses, the CES-D was used as a continuous variable.

\section{Lifestyle}

The number of alcohol consumptions was categorized into three categories: 0 alcoholic drinks per day ('none' group), 1-2 alcoholic drinks for men and 1 alcoholic drink for women per day ('minimal' group) or $>2$ alcohol drinks for men and $>1$ alcohol drink for women per day ('moderate' group) [8]. Smoking was dichotomized in 'yes (or stopped within one year)' or 'no'.

For the assessment of physical activity, the LASA Physical Activity Questionnaire (LAPAQ) was used addressing walking outdoors, bicycling, light household, heavy household, and two sports activities [21]. The subjects are asked how often and how long they carried out these activities in the past 2 weeks. In the analyses, total physical activity in minutes per day was used as a continuous variable.

\section{Cognitive outcome measures}

Two different neuropsychological tests were used as outcome measures: The Mini-Mental State Examination (MMSE) and 15 Words Test (15WT). The MMSE is the most used screening instrument for global cognitive dysfunction [22]. The score ranges from 0 to 30 points, with higher scores indicating better cognitive functioning. The 15WT is the Dutch version of the Auditory Verbal Learning Test [23]. Fifteen words have to be learned over five trials. In LASA the $15 \mathrm{WT}$ is restricted to three trials due to a limitation in time. In this study we used the maximum immediate recall score and delayed recall score, both ranging from 0 to 15 words. The delayed recall was assessed after 20 minutes of distraction.

\section{Statistical analyses}

\section{Spline regression analyses}

Former studies, have shown that the association between age and cognition is nonlinear [24]. Linear regression techniques are therefore not sufficient enough to estimate this association and spline regression analyses are indicated to fit the nonlinear longitudinal associations between age and the cognitive outcome measures more precisely (Additional file 2: Spline regression analyses) [25]. To achieve the best fit of the data with a spline regression model, either linear or cubic splines can be used. Based on the likelihood-ratio (LR) test we determined which of these two types of splines showed a better fit with our data. We examined the positions where the splines join smoothly together, referred to as knots in spline regression analyses. We identified the optimal position of the knots by testing both a model with one and two knots and moving those 5 years up and down. The ages that corresponded to the position of the knots were used to separate our sample into different age groups, to facilitate interpretation of results. Lastly, for all the different risk factors and outcome measures we compared our final model with a linear regression model without splines to test whether the model with splines showed a better fit (based on the LR test).

\section{Differences between age groups at baseline}

Statistically significant differences in baseline characteristics between the age groups were determined by using ANOVA for continuous variables, chi-square for categorical variables and Kruskal-Wallis test for skewed variables. Mixed model analysis was used to determine the difference in cognitive test score change per year in the age groups.

\section{Association of risk factors with cognitive outcome measures}

We performed three different analyses to determine the association of the risk factors (measured at baseline or, for hypertension and some biomaterial measurements, in the second cycle) with the cognitive outcome measures. In all these analyses, splines (determined as described in 2.3.1) were added to the model to estimate the association between age and the cognitive outcome measure. First, we determined the association of the risk factors with the three cognitive outcome measures in the total sample by using a linear mixed model (including a random intercept and fixed slopes). Secondly, we added the interaction of the risk factors with the splines to the analyses to assess the age dependency of the risk factors. Because the splines represent different age groups, a significant interaction means that the association of that risk factor with the cognitive outcome measure is different between age groups. If this interaction was statistically significant for a categorized risk factor, we visualized the association in a figure. Lastly, we determined the association coefficient per age group of the risk factors with the cognitive outcome measures. This last step helps us to interpret the results we found with the interaction analyses (we also performed these analyses for the risk factors that did not show a significant interaction). All the analyses were adjusted for sex and education (in years).

\section{Selection during follow-up}

To determine whether there was a selection towards healthier subjects during follow-up, we determined the 
baseline values of the risk factors and cognitive outcome measures of the subjects that were present in the sample during each LASA cycle. Decreasing baseline values during follow-up would be indicative of selection towards healthier subjects.

\section{Statistical software}

The spline regression analyses were performed with the statistical software $\mathrm{R}$ version 3.2.5 (http://www.r-project.org). The statistical significance of the association of the risk factor with cognitive decline per age group was determined with Stata version 15. The differences in baseline characteristics between the three age groups were analyzed with SPSS Statistics version 22. The level of significance was set to $p=0.05$.

\section{Results}

We included 2527 subjects ( $51.2 \%$ women) who were on average 70.1 (SD: 8.8, range: 54.8-85.6) years at baseline and had 9.1 (SD: 3.4) years of education (Table 1). Median follow-up was 9.1 (IQR: 3.2-19.0) years.

\section{Determination of the best-fitted spline regression model}

For the longitudinal associations between the cognitive outcome measures and age in the total group, a linear spline regression model showed a better fit then a cubic spline regression model. A model with two knots placed at the ages 70 and 80 years showed the best fit for the three outcome measures, dividing the sample in three age groups: $\leq 70$ years (young-old subjects), $>70-80$ years (old subjects) and $>80$ years (oldest-old subjects) (Fig. 1). The spline regression model showed a better fit then a linear regression model without splines for all the different associations.

\section{Differences between age groups at baseline}

Most characteristics differed between age groups (Table 1). Years of education, follow-up time, scores on the cognitive tests, total and LDL cholesterol levels, alcohol use, smoking and physical activity all decreased with age. The level of homocysteine and inflammation markers and the presence of cardiovascular comorbidities and depressive symptoms increased with age.

\section{Association of risk factors with cognitive decline in the total sample}

In the total sample, the presence of high homocysteine levels, history of stroke and depressive symptoms were associated with more decline in MMSE and the 15WT (Table 2). Alcohol use was associated with less decline in MMSE and the 15WT. The presence of APOE $\varepsilon 4$ was associated with more decline in MMSE and a history of DM with more decline in the 15WT. Cholesterol levels, hypertension, history of MI, inflammation markers, smoking and physical activity were not associated with cognitive decline.

\section{Age dependency of risk factors}

The association of LDL cholesterol, homocysteine, hypertension, history of stroke, depressive symptoms, IL-6, ACT, alcohol use and smoking with cognitive decline differed between the age groups (Table 3 and Fig. 2). The presence of APOE $\varepsilon 4$, total and HDL cholesterol level, a history of DM or MI, CRP level and physical activity did not show an age effect. In general, the regression coefficient changed from a negative association in the young-old and old subjects to a positive association in the oldest-old subjects. This means that on top of the decline in MMSE and 15WT as visualized in Fig. 1, the presence of these risk factors was associated with more decline in MMSE or 15WT in the young-old and old subjects and less decline in MMSE or 15WT in the oldest-old subjects. If we determined the association of the age-dependent risk factors with cognitive decline per age group, we found that hypertension, high IL-6 levels, and alcohol use were significantly associated with less cognitive decline in the oldest-old subjects (Table 3 and Additional file 1: Tables S5-S6). Smoking was significantly associated with more memory decline in the young-old subjects and high LDL cholesterol with more MMSE decline in the young-old subjects (Table 3 and Additional file 1: Table S6).

Post-hoc analyses with hypertension defined by the measured high blood pressure only or the use of antihypertensive medication only, yielded similar results (Additional file 1: Table S3).

\section{Selection during follow-up}

Subjects who were retained in the later LASA cycles had a lower age, higher level of education, higher scores on the cognitive outcome measures, higher cholesterol levels, less comorbidities, lower levels of inflammation markers, higher alcohol use, lower levels of smoking and had a higher level of physical activity at baseline compared to subjects who dropped out during follow-up (Additional file 1: Table S4).

\section{Discussion}

This study showed that the association of LDL cholesterol, homocysteine, hypertension, history of stroke, depressive symptoms, IL-6, ACT, alcohol use and smoking with cognitive decline was age-dependent. In general, these risk factors were associated with more cognitive decline in the young-old and old subjects and less cognitive decline in the oldest-old subjects. APOE $\varepsilon 4$ genotype and DM were negatively associated with cognitive decline regardless of age. 
Table 1 Baseline characteristics of subjects in the total sample

\begin{tabular}{|c|c|c|c|c|c|}
\hline Characteristic & Total sample & $\leq 70$ years & $>70-80$ years & $>80$ years & $P$-value \\
\hline Sample size ${ }^{a}$ & 2527 & 1292 & 794 & 441 & \\
\hline Age, y & $70.1(8.8)$ & $62.6(4.2)$ & $75.5(2.9)$ & $82.6(1.5)$ & \\
\hline Female, \% & 51.2 & 52.3 & 50.0 & 50.3 & 0.48 \\
\hline Education, y & $9.1(3.4)$ & $9.5(3.3)$ & $8.7(3.2)$ & $8.6(3.7)$ & $<0.01$ \\
\hline Follow-up, y (median, IQR) & $9.1(3.2-19.0)$ & $13.3(8.9-19.2)$ & $6.2(3.0-13.0)$ & $4.9(3.3-8.9)$ & $<0.01$ \\
\hline MMSE, points (median, IQR) & $28(27-29)$ & $29(26-30)$ & $28(24-30)$ & $27(24-30)$ & $<0.01$ \\
\hline Change in MMSE per year (SE) & $-0.11(0.00)$ & $-0.06(0.00)$ & $-0.18(0.01)$ & $-0.25(0.02)$ & $<0.01$ \\
\hline 15WT immediate recall, words & $8.1(2.5)$ & $8.9(2.3)$ & $7.6(2.4)$ & $6.3(2.1)$ & $<0.01$ \\
\hline Change in $15 \mathrm{WT}$ immediate recall per year (SE) & $-0.11(0.00)$ & $-0.07(0.00)$ & $-0.11(0.01)$ & $-0.07(0.02)$ & $<0.01$ \\
\hline 15WT delayed recall, words & $5.3(2.7)$ & $6.2(2.6)$ & $4.7(2.5)$ & $3.5(2.2)$ & $<0.01$ \\
\hline Change in $15 \mathrm{WT}$ delayed recall per year (SE) & $-0.11(0.00)$ & $-0.05(0.01)$ & $-0.10(0.01)$ & $-0.06(0.02)$ & $<0.01$ \\
\hline $\mathrm{APOE} \varepsilon 4, \%^{\mathrm{c}}$ & 26.3 & 27.8 & 25.2 & 23.6 & 0.29 \\
\hline Total cholesterol, mmol/L & $5.7(1.0)$ & $5.9(1.0)$ & $5.6(1.0)$ & $5.3(1.1)$ & $<0.01$ \\
\hline LDL cholesterol, mmol/L & $3.7(1.0)$ & $3.8(0.9)$ & $3.6(0.9)$ & $3.4(1.1)$ & $<0.01$ \\
\hline HDL cholesterol, mmol/L & $1.3(0.4)$ & $1.3(0.4)$ & $1.4(0.4)$ & $1.3(0.4)$ & 0.48 \\
\hline Homocysteine, mmol/L & $14.5(6.1)$ & $13.5(5.2)$ & $14.7(5.0)$ & $17.2(9.2)$ & $<0.01$ \\
\hline Vitamin B12, pMol/L (median, IR) & $266(212-333)$ & $268(219-335)$ & $264(213-331)$ & $249(196-333)$ & 0.12 \\
\hline Hypertension, \% & 76.7 & 72.9 & 82.2 & 73.7 & $<0.01$ \\
\hline Myocardial infarction, \% & 8.8 & 6.4 & 11.1 & 11.7 & $<0.01$ \\
\hline Diabetes mellitus, \% & 7.0 & 4.3 & 8.6 & 12.3 & $<0.01$ \\
\hline Stroke, \% & 4.4 & 1.6 & 6.7 & 8.7 & $<0.01$ \\
\hline CES-D total score (median, IQR) & $5(2-11)$ & $5(2-9)$ & $6(3-11)$ & $7(3-12)$ & $<0.01$ \\
\hline IL-6, pg/ml (median, IQR) & $1.4(0.6-2.5)$ & $1.3(0.6-2.4)$ & $1.6(0.7-2.7)$ & $1.8(1.0-3.1)$ & $<0.01$ \\
\hline CRP, ug/ml (median, IQR) & $2.2(1.0-4.7)$ & $2.0(0.9-3.9)$ & $2.5(1.3-5.6)$ & $2.8(1.4-5.7)$ & $<0.01$ \\
\hline $\mathrm{ACT}, \%$ of $\mathrm{NHP}$ & $173.6(57.6)$ & $169.6(53.0)$ & $179.3(66.1)$ & $177.2(54.1)$ & 0.01 \\
\hline \multicolumn{6}{|l|}{ Alcohol consumption, \% } \\
\hline None & 20.1 & 15.6 & 25.3 & 24.7 & $<0.01$ \\
\hline Minimal $^{d}$ & 20.6 & 17.8 & 23.6 & 23.6 & \\
\hline Moderate $^{e}$ & 59.4 & 66.6 & 51.1 & 51.7 & \\
\hline Smokers, \% & 24.6 & 30.4 & 23.1 & 19.8 & $<0.01$ \\
\hline Total physical activity, min per day & $169.2(114.2)$ & $188.4(121.3)$ & $160.0(103.7)$ & $126.8(95.3)$ & $<0.01$ \\
\hline
\end{tabular}

15WT 15 Words Test, $A C T$ a1-antichymotrypsin, APOE apolipoprotein E, CES-D Center for Epidemiologic Studies Depression scale, CRP C-reactive protein, HDL HighDensity Lipoprotein, IL-6 interleukin-6, IQR interquartile range, LASA Longitudinal Aging Study Amsterdam, LDL Low-Density Lipoprotein, MMSE Mini-Mental State Examination, NHP normal human plasma, SE standard error

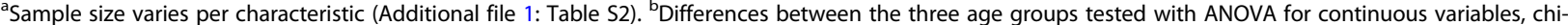
square for categorical variables, Kruskal-Wallis test for non-parametric variables and mixed model analysis for the change in cognitive test scores per year. ${ }^{c}$ Percentage of subjects with an apolipoprotein E $\varepsilon 4$ isoform as proxy for an APOE $\varepsilon 4$ allele. ${ }^{d}$ Women:1 drink/day, men: $1-2$ drinks/day. ${ }^{e}$ Women: $>1$ drink/day, men: $>2$ drinks/day. Values are means (SD) unless stated otherwise

Before we discuss these findings in more detail, it should be noted that the baseline age of the subjects with follow-up was lower than that of the subjects who were lost to follow up. The subjects with follow-up also showed a better overall health with less comorbidity. The selection towards younger and healthier subjects at follow-up may explain why the negative impact of the risk factors was strongest in younger subjects. However, it does not explain why these risk factors became protective at higher age. We reduced the potential selection bias by combining baseline data and follow-up data across the age span in the spline regression model. In this way follow-up data of the selected younger, healthier subjects (Additional file 1: Table S4) were combined with baseline data of the older, less healthy subjects (Table 1).

In addition, most subjects dropped out of the study because of mortality [15]. A comparison of one-year 

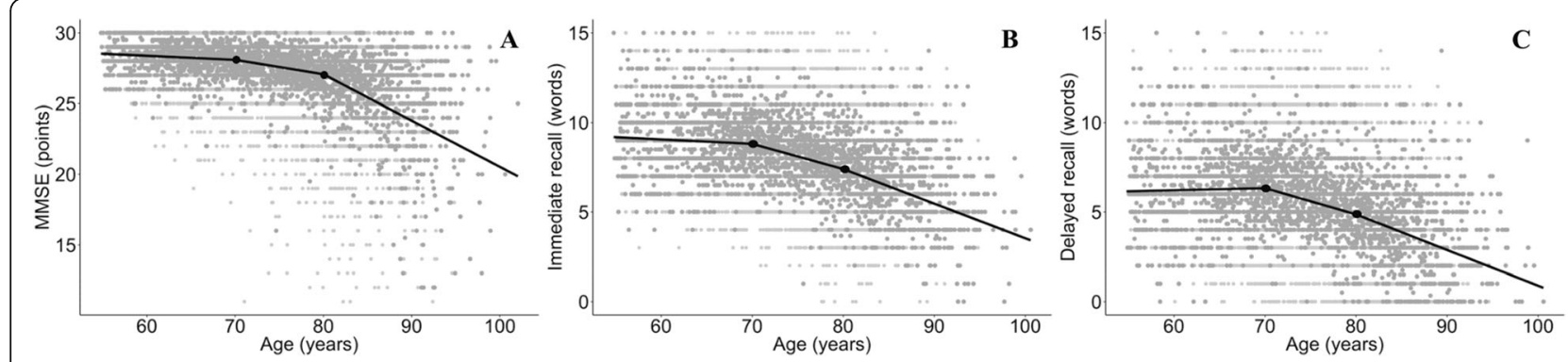

Fig. 1 Association between age and Mini-Mental State Examination (MMSE, figure $\mathbf{a})$ and 15 Words Test (15WT, figure $\mathbf{b}$ and $\mathbf{c}$ ). The grey dots represent all the longitudinal data points. The lines represent the splines with the two knots (black dots) at the ages 70 and 80 years

mortality rates of LASA subjects with those in the general Dutch population showed that mortality in the LASA subjects was slightly higher than in the general population, but that this difference exceeded $1 \%$ only in women aged 80-85 years (unpublished data). Therefore, it may not necessarily affect the generalizability of our findings.

\section{APOE genotype}

Our finding that the APOE $\varepsilon 4$ genotype increases the risk for cognitive decline regardless of age is at odds with some earlier studies that reported a decrease of risk for dementia with age $[11,26]$ but is consistent with others [27]. Differences between studies may be explained by differences in selection of subjects (normal cognition or $\mathrm{MCI}$ ) and outcome measure (progression to dementia or cognitive decline).

\section{Cardiovascular factors}

Most cardiovascular factors were associated with less decline at higher age than at younger age, which is in line with previous studies [12, 28]. High cholesterol in late-life can be an indicator of a better nutritional status and a better overall health and therefore associated with less cognitive decline [28, 29]. Additionally, cholesterol synthesis is thought to decrease with aging, but only in $\mathrm{AD}$ patients and not in subjects with a normal cognition [30]. The association of low cholesterol with more

Table 2 The association of risk factors with cognitive decline in the total sample

\begin{tabular}{|c|c|c|c|c|c|c|c|c|c|}
\hline \multirow[t]{2}{*}{ Risk factor } & \multicolumn{3}{|l|}{ MMSE } & \multicolumn{3}{|c|}{ 15WT immediate recall } & \multicolumn{3}{|c|}{ 15WT delayed recall } \\
\hline & B & SE & $P$-value & $B$ & SE & P-value & B & SE & $P$-value \\
\hline APOE $\varepsilon 4$ & -0.25 & 0.24 & $<0.01$ & -0.07 & 1.27 & 0.49 & -0.09 & 1.44 & 0.42 \\
\hline Total cholesterol & 0.05 & 0.17 & 0.34 & 0.09 & 1.22 & 0.11 & 0.08 & 1.39 & 0.21 \\
\hline LDL cholesterol & 0.05 & 0.17 & 0.41 & 0.08 & 1.24 & 0.20 & 0.08 & 1.41 & 0.25 \\
\hline HDL cholesterol & 0.16 & 0.16 & 0.20 & 0.23 & 1.21 & 0.08 & 0.07 & 1.42 & 0.67 \\
\hline Homocysteine & -0.05 & 0.12 & $<0.01$ & -0.05 & 1.16 & $<0.01$ & -0.06 & 1.33 & $<0.01$ \\
\hline Hypertension & 0.13 & 0.28 & 0.26 & -0.02 & 1.26 & 0.88 & -0.03 & 1.43 & 0.85 \\
\hline DM & -0.16 & 0.29 & 0.25 & -0.42 & 1.28 & 0.01 & -0.42 & 1.45 & 0.04 \\
\hline Ml & 0.05 & 0.29 & 0.69 & 0.08 & 1.28 & 0.58 & 0.22 & 1.48 & 0.21 \\
\hline Stroke & -0.40 & 0.28 & 0.02 & -0.55 & 1.27 & 0.01 & -0.58 & 1.44 & 0.02 \\
\hline Depressive symptoms & -0.01 & 0.33 & $<0.01$ & -0.02 & 1.33 & $<0.01$ & -0.02 & 1.50 & $<0.01$ \\
\hline CRP & 0.00 & 0.27 & 0.57 & -0.01 & 1.27 & 0.15 & -0.01 & 1.45 & 0.47 \\
\hline IL-6 & 0.01 & 0.26 & 0.46 & 0.01 & 1.27 & 0.72 & 0.02 & 1.44 & 0.42 \\
\hline ACT & 0.00 & 0.27 & 0.68 & 0.00 & 1.27 & 0.74 & 0.00 & 1.45 & 0.87 \\
\hline Alcohol $^{a}$ : minimal ${ }^{b}$ & 0.27 & 0.10 & $<0.01$ & 0.35 & 0.12 & $<0.01$ & 0.35 & 0.14 & 0.01 \\
\hline Alcohol $^{a}$ : moderate ${ }^{c}$ & 0.24 & 0.08 & $<0.01$ & 0.41 & 0.10 & $<0.01$ & 0.42 & 0.12 & $<0.01$ \\
\hline Smoking & -0.09 & 0.25 & 0.26 & -0.16 & 1.27 & 0.09 & -0.03 & 1.46 & 0.77 \\
\hline Physical activity & 0.00 & 0.30 & 0.78 & 0.00 & 1.28 & 0.59 & 0.00 & 1.45 & 0.24 \\
\hline
\end{tabular}

$B^{\prime} s$ are determined by linear mixed models in combination with splines and adjusted for sex and education

15WT 15 Words Test, ACT a1-antichymotrypsin, APOE apolipoprotein E, CRP C-reactive protein, DM Diabetes mellitus, HDL High-Density Lipoprotein, IL-6 interleukin-6, LDL Low-Density Lipoprotein, MI Myocardial infarction, MMSE Mini-Mental State Examination

${ }^{\mathrm{a}} \mathrm{No}$ alcohol use is reference group. ${ }^{b}$ Women:1 drink/day, men: $1-2$ drinks/day. ${ }^{C}$ Women: $>1$ drink/day, men: $>2$ drinks/day 
Table 3 The association of risk factors with cognitive decline per age group

\begin{tabular}{|c|c|c|c|c|c|c|c|c|c|}
\hline \multirow[t]{2}{*}{ Risk factor } & \multicolumn{3}{|l|}{ MMSE } & \multicolumn{3}{|c|}{ 15WT immediate recall } & \multicolumn{3}{|c|}{ 15WT delayed recall } \\
\hline & $\leq 70$ & $>70-80$ & $>80$ & $\leq 70$ & $>70-80$ & $>80$ & $\leq 70$ & $>70-80$ & $>80$ \\
\hline APOE $\varepsilon 4$ & 0.72 & -4.24 & -10.17 & -0.65 & -3.85 & -5.32 & 0.18 & -4.15 & -8.52 \\
\hline Total cholesterol & -3.37 & -0.10 & 1.26 & -1.08 & -1.09 & 1.53 & 0.97 & -1.53 & 1.27 \\
\hline LDL cholesterol & $-5.40^{*}$ & 0.66 & $1.83^{*}$ & -0.18 & $-1.92^{*}$ & $1.96^{*}$ & 0.68 & -2.62 & 1.41 \\
\hline HDL cholesterol & 7.35 & -2.17 & -1.07 & -1.84 & 2.64 & 0.50 & 6.21 & 1.45 & 2.97 \\
\hline Homocysteine & 0.39 & -0.40 & -0.92 & -0.39 & -0.21 & 0.13 & $-0.76^{*}$ & -0.08 & $0.13^{*}$ \\
\hline Hypertension & 0.04 & $-2.14^{*}$ & $6.52^{*}$ & $-5.65^{*}$ & -0.22 & $5.06^{*}$ & -4.04 & 3.54 & 0.43 \\
\hline DM & -0.95 & -0.46 & -9.32 & -0.11 & -3.49 & -0.29 & -1.68 & -5.44 & 4.90 \\
\hline Ml & 2.42 & -1.59 & 2.23 & 0.41 & 1.93 & 5.64 & 4.90 & 0.62 & 10.14 \\
\hline Stroke & 1.25 & $-9.16^{*}$ & $9.16^{*}$ & 2.60 & -0.34 & 6.70 & 0.79 & -1.58 & -4.30 \\
\hline Depressive symptoms & -0.07 & 0.02 & -0.06 & -0.21 & 0.00 & 0.07 & $-0.11^{*}$ & -0.23 & $0.21^{*}$ \\
\hline CRP & -0.18 & 0.18 & 0.19 & -0.21 & 0.03 & 0.19 & -0.19 & -0.11 & 0.16 \\
\hline IL-6 & $0.02^{*}$ & $-0.10^{*}$ & $1.31 *$ & 0.27 & -0.11 & 0.64 & 0.31 & -0.03 & 0.88 \\
\hline ACT & 0.00 & 0.01 & 0.06 & -0.01 & -0.01 & 0.03 & $-0.01^{*}$ & -0.04 & $0.03^{*}$ \\
\hline Alcohol $^{a}$ : minimal ${ }^{b}$ & $2.82^{*}$ & -0.26 & $7.93^{*}$ & 3.19 & -3.09 & -0.31 & 5.91 & -3.03 & 0.25 \\
\hline Alcohol $^{a}$ : moderate ${ }^{c}$ & 0.78 & -0.95 & 3.48 & 2.85 & -3.51 & 1.42 & 3.19 & -0.47 & -0.69 \\
\hline Smoking & -0.30 & -1.05 & -5.15 & $-5.84^{*}$ & $0.53^{*}$ & -1.13 & -4.52 & -2.88 & -0.61 \\
\hline Physical activity & 0.00 & 0.00 & 0.02 & -0.01 & 0.01 & 0.00 & 0.01 & 0.01 & 0.00 \\
\hline
\end{tabular}

Shown are beta's (multiplied by 100) of the associations of a risk factor with cognitive decline within each age group. They show the extra cognitive decline (next to the overall cognitive decline as visualized in Fig. 1) per age group in the presence of a risk factor. A negative beta indicates that a unit increase in the risk factor is associated with more cognitive decline. Bold beta's indicate a significant $(p<0.05)$ association with cognitive decline in that age group (in Additional file 1: Tables S5-S7 we present the standard errors and $p$-values corresponding to the beta's in this table per age group)

15WT 15 Words Test, ACT a1-antichymotrypsin, IL-6 interleukin-6, LDL Low-Density Lipoprotein, MMSE Mini-Mental State Examination

${ }^{*}$ Association of risk factor with MMSE or $15 \mathrm{WT}$ decline is significantly different between these two age groups. In case of three *: difference is significant between $\leq 70$ and $>80$ years old group and between $>70-80$ and $>80$ years old group. Beta's are determined by linear mixed models in combination with splines and adjusted for sex and education

${ }^{a}$ No alcohol use is reference group. ${ }^{b}$ Women:1 drink/day, men: 1-2 drinks/day. ${ }^{c}$ Women: $>1$ drink/day, men: $>2$ drink/day

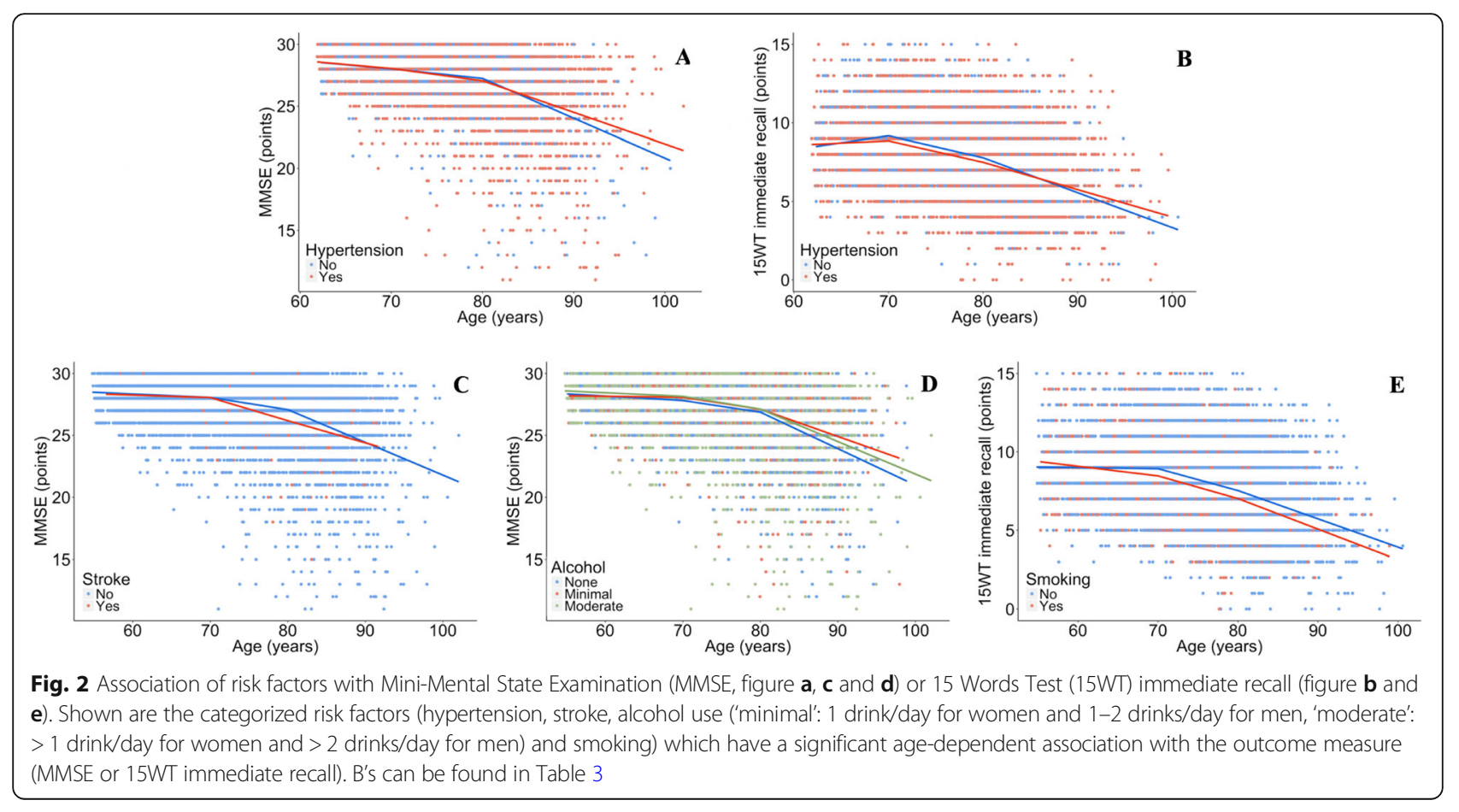


cognitive decline in the oldest-old subjects might therefore be an expression of underlying AD pathology.

Hypertension may prevent cognitive decline at old age by ensuring the cerebral blood flow [31, 32]. On the other hand, low blood pressure can be a consequence of neurodegenerative disease and therefore an early sign of dementia onset, although this is an aspect which one can also expect in the young-old subjects [33,34].

\section{Depressive symptoms}

Earlier studies have shown that depressive symptoms are an important risk factor for cognitive decline and dementia, also among the oldest-old subjects $[7,35]$. We replicated this finding for the total sample but also found that the association of depressive symptoms with memory decline was less in the oldest-old group than in the young-old group. This may be explained by the fact that older subjects score higher on the CES-D questionnaire for reasons other than depression, such as somatic morbidity [36]. In line with this explanation, earlier cross-domain latent growth models on LASA data demonstrated that delayed recall was associated with increasing levels of depressed affect, but not with depressive somatic symptoms [37].

\section{Inflammation markers}

Inflammation has been described as an important mechanism underlying cognitive decline but most of these studies were performed in younger subjects [38]. We did not find an association of inflammation markers with cognitive decline in the total sample but noted differences between age groups showing that higher IL- 6 and ACT levels were associated with less cognitive decline in subjects aged 80 years and older compared to younger subjects. Potentially, higher inflammation markers in older subjects are a sign of a better inflammatory response and therefore related to better overall health and cognitive functioning.

\section{Lifestyle factors}

Minimal and moderate alcohol use were positively associated with decline in MMSE and memory functioning compared to no alcohol use, which is in line with earlier studies $[3,8]$. We found that the positive association of alcohol use with cognition was strongest in subjects aged 80 years and older. In this age group, no alcohol use is frequently related to poor physical functioning. Therefore, the negative association of no alcohol use with cognitive decline is potentially an indirect effect [39].

In accordance with our findings, a meta-analysis in 2015 showed that the negative association of smoking with cognitive decline is decreasing with age [40]. Survival bias and the presence of competing risks are probably the most important phenomena to explain this finding [40-42].

In contrast to a meta-analysis of observational studies, we did not find an association between physical activity and cognitive decline [43]. However, a meta-analysis of intervention studies on the effect of aerobic exercise on cognitive decline in cognitively normal subjects did not find an effect [44]. We found that in the oldest age group less physical activity was associated with more cognitive decline, but because the over age interaction effect was not statistically significant, this finding should be interpreted cautiously.

\section{Cognitive outcome measures}

We used different cognitive tests as outcome measure (MMSE, 15WT total and delayed recall) and in the total sample, most variables showed similar findings for the different outcome measures and the course of the tests was very similar with age (Table 2 and Fig. 1). However, we also found different results for the different outcomes, which may be explained by the fact that the tests measure different disease processes; memory decline is presumed to be an early marker of Alzheimer's disease and decline in MMSE can be caused by a broader range of diseases [45].

\section{Strengths and limitations}

This is the first study that analyzes the influence of age on the association of different types of risk factors with cognitive decline in one prospective cohort study. Earlier studies have indicated age differences but never studied the various risk factors in one cohort. Additionally, the use of a nonlinear analyze technique, is an important added value of this study to earlier literature. A limitation of this study is that we did not have data about the presence of cardiovascular risk factors, such as hypertension and high cholesterol, before age 55 years. Therefore, we could not discriminate between a high blood pressure and cholesterol emerging at high age or already present at younger ages. While we tested many risk factors with different outcomes at the same time, this also increased the risk of false-positive findings. However, we decided not to correct for multiple testing given the exploratory nature of the study and the increased risk of missing important findings when applying Bonferroni adjustments (type II errors) [46, 47]. For simplification purposes, we describe our results in relation to cognitive decline, although we only used the MMSE and 15WT to assess cognition. It therefore needs to be noted that these results cannot automatically be extrapolated to other forms of cognition. In addition, the 15WT is a test for verbal episodic memory and does not assess other types of memory such as visual and semantic memory. 


\section{Scientific and clinical implications}

With this study we showed that age needs to be taken into consideration when studying risk factors for cognitive decline. It is not only needed to add age as a confounder but especially as an effect modifier to analyses as it changes the relation between a risk factor and cognitive decline. From a clinical perspective, these results suggest that different preventive strategies might be effective in young-old versus oldest-old subjects. Antihypertensive and cholesterol lowering medication might not be appropriate for the oldest-old subjects who develop hypertension and hypercholesterolemia at a high age.

\section{Conclusions}

The associations of LDL cholesterol, homocysteine, hypertension, history of stroke, depressive symptoms, IL-6, $\mathrm{ACT}$, alcohol use and smoking with cognitive decline were different per age group. They were all less strongly associated with cognitive decline in the older subjects compared to younger subjects. Selection towards healthier subjects during follow-up need to be considered as possible explanation but also risk factor specific considerations, such as ensuring the cerebral blood flow in case of hypertension, need to be taken into account. These age differences are important when applying preventive strategies to avert cognitive decline.

\section{Additional files}

Additional file 1: Additional tables about sample sizes, the association of hypertension with cognitive decline separately for use of antihypertensive medication and blood pressure, baseline characteristics per LASA cycle and the associations of the risk factors with the three cognitive tests in the three age groups. (DOCX $72 \mathrm{~kb}$ )

Additional file 2: Additional explanation about the spline regression analyses. (DOCX 375 kb)

\section{Abbreviations \\ 15WT: 15 Words Test; ACT: A1-antichymotrypsin; AD: Alzheimer's dementia; APOE: Apolipoprotein E; CES-D: Center for Epidemiologic Studies Depression scale; CRP: C-reactive protein; DM: Diabetes mellitus; HDL cholesterol: High-Density Lipoprotein cholesterol; IL-6: Interleukin-6; IQR: Interquartile range; LAPAQ: LASA Physical Activity Questionnaire; LASA: Longitudinal Aging Study Amsterdam; LDL cholesterol: Low-Density Lipoprotein cholesterol; LR: Likelihood-ratio; MAAS: Maastricht Aging Study; MI: Myocardial infarction; MMSE: Mini-Mental State Examination; NHP: Normal human plasma; SD: Standard deviation; SE: Standard error; VLDL cholesterol: Very-low-density lipoprotein cholesterol; VUmc: VU University Medical Center}

\section{Funding}

This work has received support from the EU/EFPIA Innovative Medicines Initiative Joint Undertaking EMIF grant agreement no. 115372. The Longitudinal Aging Study Amsterdam (LASA) is primarily funded by the Dutch Ministry of Health, Welfare and Sports and the VU University Amsterdam.

\section{Availability of data and materials}

The datasets used and/or analyzed during the current study can be requested through http://www.lasa-vu.nl/data/availability_data/availability_data.htm.

\section{Authors' contributions}

$\mathrm{MH}$ and $\mathrm{HCC}$ are both involved in the design of the Longitudinal Aging Study Amsterdam and are responsible for the data collection. PJV created the concept for this manuscript. MWH and NL performed the statistical analyses. NL wrote the first and final drafts of the manuscript. MWH, HCC, $\mathrm{MH}, \mathrm{ABM}$ and PJV contributed to and approved the final version.

\section{Ethics approval and consent to participate}

All subjects gave written informed consent and the study was approved by the Ethical Review Board of the VU University Medical Center (VUmc) and conducted according to the principles of the Helsinki declaration.

\section{Consent for publication}

Not applicable.

\section{Competing interests}

PJV is currently acting as a Section Editor for BMC Geriatrics. The other authors declare that they have no competing interests.

\section{Publisher's Note}

Springer Nature remains neutral with regard to jurisdictional claims in published maps and institutional affiliations.

\section{Author details}

${ }^{1}$ Alzheimer Center Amsterdam, Department of Neurology, Amsterdam Neuroscience, Vrije Universiteit Amsterdam, Amsterdam UMC, PO Box 7057 1007, MB, Amsterdam, the Netherlands. 'Department of Epidemiology and Biostatistics, Amsterdam Public Health Research Institute, Vrije Universiteit Amsterdam, Amsterdam, the Netherlands. ${ }^{3} \mathrm{GGZ}$ inGeest / Department of Psychiatry, Amsterdam Public Health Research Institute, Vrije Universiteit Amsterdam, Amsterdam, the Netherlands. ${ }^{4}$ Department of Sociology, Vrije Universiteit Amsterdam, Amsterdam, the Netherlands. ${ }^{5}$ Department of Medicine and Aged Care, @AgeMelbourne, Royal Melbourne Hospital, University of Melbourne, Melbourne, Australia. ${ }^{6}$ Department of Human Movement Sciences, @AgeAmsterdam, Faculty of Behavioural and Movement Sciences, Vrije Universiteit Amsterdam, Amsterdam, the Netherlands. ${ }^{7}$ Department of Psychiatry \& Neuropsychology, School for Mental Health and Neuroscience, Maastricht University, Maastricht, the Netherlands.

Received: 4 May 2018 Accepted: 7 August 2018

Published online: 20 August 2018

\section{References}

1. World Health Organization and Alzheimer's Disease International. Dementia - A public health priority. 2012.

2. Kramer AF, Colcombe SJ, McAuley E, Scalf PE, Erickson KI. Fitness, aging and neurocognitive function. Neurobiol Aging. 2005;26:124-7. https://doi.org/10. 1016/j.neurobiolaging.2005.09.009.

3. Baumgart M, Snyder HM, Carrillo MC, Fazio S, Kim H, Johns H. Summary of the evidence on modifiable risk factors for cognitive decline and dementia: a population-based perspective. Alzheimers Dement. 2015;11:1-9. https://doi.org/10.1016/j.jalz.2015.05.016.

4. Smith RG, Todd S, Passmore PA. Chronic medical disease and cognitive aging: toward a healthy body and brain. Inflammation and Cognitive Decline. Edited by Kristine Yaffe. Can Geriatr J. 2013;16(3):143-44.

5. Bretsky P, Guralnik JM, Launer L, Albert M, Seeman TE. The role of APOEepsilon4 in longitudinal cognitive decline: MacArthur studies of successful aging. Neurology. 2003;60:1077-81. https://doi.org/10.1212/01.WNL.00000 55875.26908 .24

6. Cheng G, Huang C, Deng H, Wang H. Diabetes as a risk factor for dementia and mild cognitive impairment: a meta-analysis of longitudinal studies. Intern Med J. 2012;42:484-91. https://doi.org/10.1111/j.1445-5994.2012.02758.x.

7. Van Den Kommer TN, Comijs HC, Aartsen MJ, Huisman M, Deeg DJH, Beekman ATF. Depression and cognition: how do they interrelate in old age? Am J Geriatr Psychiatry. 2013;21:398-410. https://doi.org/10.1016/j.jagp.2012.12.015.

8. Anstey KJ, Mack HA, Cherbuin N. Alcohol consumption as a risk factor for dementia and cognitive decline: Meta-analysis of prospective studies. Am J Geriatr Psychiatry. 2009;17:542-55. https://doi.org/10.1097/JGP.0b013e3181 a2fd07. 
9. Anstey KJ, Lipnicki DM, Low L-F. Cholesterol as a risk factor for dementia and cognitive decline: a systematic review of prospective studies with Meta-analysis. Am J Geriatr Psychiatry. 2008;16:343-54. https://doi.org/10. 1097/01.JGP.0000310778.20870.ae.

10. Bullain SS, Corrada MM. Dementia in the oldest old. Continuum (Minneap Minn). 2013:19:457-69. https://doi.org/10.1212/01.CON.0000429172.27815.3f.

11. Farrer LA, Cupples LA, Haines JL, Hyman B, Kukull WA, Mayeux R, et al. Effects of age, sex, and ethnicity on the association between apolipoprotein E genotype and Alzheimer disease. A meta-analysis. APOE and Alzheimer disease Meta analysis consortium. JAMA. 1997;278:1349-56. https://doi.org/ 10.1001/jama.1997.03550160069041.

12. Qiu C, Winblad B, Fratiglioni L. The age-dependent relation of blood pressure to cognitive function and dementia. Lancet Neurol. 2005;4:487-99. https://doi.org/10.1016/S1474-4422(05)70141-1.

13. Harrison SL, Stephan BCM, Siervo M, Granic A, Davies K, Wesnes KA, et al. Is there an association between metabolic syndrome and cognitive function in very old adults? The Newcastle 85+ study. J Am Geriatr Soc. 2015;63:66775. https://doi.org/10.1111/jgs.13358.

14. van den Berg E, Biessels GJ, de Craen a J, Gussekloo J, RGJ W. The metabolic syndrome is associated with decelerated cognitive decline in the oldest old. Neurology. 2007;69:979-85. https://doi.org/10.1212/01.wnl.0000271381.30143.75.

15. Huisman M, Poppelaars J, van der Horst M, Beekman ATF, Brug J, van Tilburg TG, et al. Cohort profile: the longitudinal aging study Amsterdam. Int J Epidemiol. 2011;40:868-76. https://doi.org/10.1093/ije/dyq219.

16. Reijs BLR, Ramakers IHGB, Elias-Sonnenschein L, Teunissen CE, Koel-Simmelink M, Tsolaki M, et al. Relation of odor identification with Alzheimer's disease markers in cerebrospinal fluid and cognition. J Alzheimers Dis. 2017;60:1025-34. https://doi.org/10.3233/JAD-170564.

17. Havekes LM, De Knijff P, Beisiegel U. A rapid micromethod for apolipoprotein $E$ phenotyping directly in serum. J Lipid Res. 1987;28:455-63.

18. Friedewald WT, Levy RI, Fredrickson DS. Estimation of the concentration of low-density lipoprotein cholesterol in plasma, without use of the preparative ultracentrifuge. Clin Chem. 1972;18:499-502.

19. Kriegsman DM, Penninx BW, van Eijk JT, Boeke AJ, Deeg DJ. Self-reports and general practitioner information on the presence of chronic diseases in community dwelling elderly. A study on the accuracy of patients' self-reports and on determinants of inaccuracy. J Clin Epidemiol. 1996:49:1407-17. https:/doi.org/10.1016/S0895-4356(96)00274-0.

20. Radloff LS, Teri L. Use of the Center for Epidemiological Studies-Depression Scale with older adults. Clin Gerontol. 1986;5:119-36. https://doi.org/10. 1007/BF01537606.

21. Stel VS, Smit JH, Pluijm SMF, Visser M, Deeg DJH, Lips P. Comparison of the LASA physical activity questionnaire with a 7-day diary and pedometer. J Clin Epidemiol. 2004;57:252-8. https://doi.org/10.1016/j.jclinepi.2003.07.008.

22. Folstein MF, Folstein SE, McHugh PR. "Mini-mental state". A practical method for grading the cognitive state of patients for the clinician. J Psychiatr Res. 1975;12:189-98. https://doi.org/10.1016/0022-3956(75)90026-6.

23. Rey A. L'examen clinique en psychologie. Paris: Presses Universitaires de France; 1964.

24. Verhaeghen $P$, Salthouse TA. Meta-analyses of age-cognition relations in adulthood: estimates of linear and nonlinear age effects and structural models. Psychol Bull. 1997;122:231-49. https://doi.org/10.1037//0033-2909. 122.3.231.

25. Chen H, Zhao B, Cao G, Proges EC, O'Shea A, Woods AJ, et al. Statistical approaches for the study of cognitive and brain aging. Front Aging Neurosci. 2016:8:1-10. https://doi.org/10.3389/fnagi.2016.00176.

26. Corrada MM, Paganini-Hill A, Berlau DJ, Kawas CH. Apolipoprotein e genotype, dementia, and mortality in the oldest old: the $90+$ study. Alzheimers Dement. 2013;9:12-8. https://doi.org/10.1016/j.jalz.2011.12.004.

27. Elias-Sonnenschein LS, Viechtbauer W, Ramakers $1 H G B$, Verhey FRJ, Visser PJ. Predictive value of $\mathrm{APOE}-\varepsilon 4$ allele for progression from $\mathrm{MCl}$ to $\mathrm{AD}$-type dementia: a meta-analysis. J Neurol Neurosurg Psychiatry. 2011:82:1149-56. https://doi.org/10.1136/jnnp.2010.231555.

28. Mielke MM, Zandi PP, Sjögren M, Gustafson D, Ostling S, Steen B, et al. High total cholesterol levels in late life associated with a reduced risk of dementia. Neurology. 2005;64:1689-95. https://doi.org/10.1212/01.WNL. 0000161870.78572.A5

29. Weverling-Rijnsburger AW, Blauw GJ, Lagaay AM, Knook DL, Meinders AE, Westendorp RG. Total cholesterol and risk of mortality in the oldest old. Lancet (London, England). 1997:350:1119-23. https://doi.org/10.1016/S01406736(97)04430-9
30. van den Kommer TN, Dik MG, Comijs HC, Lutjohann D, Lips P, Jonker C, et al. The role of extracerebral cholesterol homeostasis and ApoE e4 in cognitive decline. Neurobiol Aging. 2012;33:622.e17-28. https://doi.org/10.1016/j. neurobiolaging.2011.02.019.

31. Corrada MM, Hayden KM, Paganini-Hill A, Bullain SS, DeMoss J, Aguirre C, et al. Age of onset of hypertension and risk of dementia in the oldest-old: the 90+ study. Alzheimers Dement. 2017;13:103-10. https://doi.org/10.1016/j.jalz.2016. 09.007 .

32. Kennelly SP, Lawlor BA, Kenny RA. Blood pressure and the risk for dementiaa double edged sword. Ageing Res Rev. 2009;8:61-70. https://doi.org/10. 1016/j.arr.2008.11.001.

33. Ruitenberg A, Skoog I, Ott A, Aevarsson O. Blood pressure and risk of dementia: results from the Rotterdam study and the Gothenburg H-70 study. Blood Press. 2001:33-9. https://doi.org/10.1159/000051233.

34. Skoog I, Lernfelt B, Landahl S, Palmertz B, Andreasson LA, Nilsson L, et al. 15-year longitudinal study of blood pressure and dementia. Lancet (London, England). 1996;347:1141-5. https://doi.org/10.1016/S0140-6736(96)90608-X.

35. Spira AP, Rebok GW, Stone KL, Kramer JH, Yaffe K. Depressive symptoms in oldest-old women: risk of mild cognitive impairment and dementia. Am J Geriatr Psychiatry. 2011;19:S56-7.

36. Berkman LF, Berkman CS, Kasl S, Freeman DHJ, Leo L, Ostfeld AM, et al. Depressive symptoms in relation to physical health and functioning in the elderly. Am J Epidemiol. 1986;124:372-88. https://doi.org/10.1093/ oxfordjournals.aje.a114408.

37. Brailean A, Aartsen MJ, Muniz-Terrera G, Prince M, Prina AM, Comijs HC, et al. Longitudinal associations between late-life depression dimensions and cognitive functioning: a cross-domain latent growth curve analysis. Psychol Med. 2017:47:690-702. https://doi.org/10.1017/S003329171600297X.

38. Gorelick PB. Role of inflammation in cognitive impairment: results of observational epidemiological studies and clinical trials. Ann N Y Acad Sci. 2010;1207:155-62. https://doi.org/10.1111/j.1749-6632.2010.05726.x.

39. Anstey KJ, Windsor TD, Rodgers B, Jorm AF, Christensen H. Lower cognitive test scores observed in alcohol abstainers are associated with demographic personality, and biological factors: the PATH through life project. Addiction. 2005:100:1291-301. https://doi.org/10.1111/j.1360-0443.2005.01159.x.

40. Zhong G, Wang Y, Zhang Y, Guo JJ, Zhao Y. Smoking is associated with an increased risk of dementia: a meta-analysis of prospective cohort studies with investigation of potential effect modifiers. PLoS One. 2015;10 https://doi.org/10.1371/journal.pone.0118333.

41. Chang C-CH, Zhao Y, Lee C-W, Ganguli M. Smoking, death, and Alzheimer's disease: a case of competing risks. Alzheimer Dis Assoc Disord. 2012;26: 300-6. https://doi.org/10.1097/WAD.0b013e3182420b6e.

42. Hernán MA, Alonso ALG. Cigarette smoking and dementia: potential selection bias in the elderly. Epidemiol (Cambridge, Mass). 2008;19:242-6. https://doi. org/10.1097/EDE.0b013e31816bbe14.

43. Blondell SJ, Hammersley-Mather R, Veerman J. Does physical activity prevent cognitive decline and dementia?: a systematic review and metaanalysis of longitudinal studies. BMC Public Health. 2014;14:1-12. https://doi. org/10.1186/1471-2458-14-510.

44. Young J, Angevaren M, Rusted J, Tabet N. Aerobic exercise to improve cognitive function in older people without known cognitive impairment (review). Cochrane Database Syst Rev. 2015:1-141. https://doi.org/10.1002/ 14651858.CD005381.pub4. www.cochranelibrary.com

45. Jansen WJ, Ossenkoppele R, Tijms BM, Fagan AM, Hansson O, Klunk WE, et al. Association of cerebral amyloid- $\beta$ aggregation with cognitive functioning in persons without dementia. JAMA Psychiatry. 2018;75:84-95. https://doi.org/10. 1001/jamapsychiatry.2017.3391.

46. Bender R, Lange S. Adjusting for multiple testing - when and how? J Clin Epidemiol. 2001:54:343-9. https://doi.org/10.1016/S0895-4356(00)00314-0.

47. Perneger TV. What's wrong with Bonferroni adjustments. BMJ. 1998;316 https://doi.org/10.1136/bmj.316.7139.1236. 